\title{
Switching Effects and Metal-Insulator Transition in Manganese Oxide
}

\author{
V. P. Malinenko, L. A. Aleshina, A. L. Pergament, and G. V. Germak
}

\begin{abstract}
MnO}_{2}$ samples are obtained by pyrolytic decomposition of manganese nitrate, and thin films of manganese oxide are also deposited by vacuum thermal evaporation and by electrochemical anodic oxidation of metal Mn. The measurements of the electrical conductivity temperature dependences reveal the change of conductivity mechanisms depending on temperature in the range of 450-25 $\mathrm{K}$ and the metal-insulator phase transition at $T \sim 80 \mathrm{~K}$ is described. The switching effects with $\mathrm{N}$-shaped (at low temperatures) and S-shaped (at room temperatures and above) $I-V$ characteristics are studied. Switching mechanisms are discussed based on metal-insulator transitions and an electrothermal instability.
\end{abstract}

Index Terms - Manganese dioxide, crystal structure, electrical switching, metal-insulator transition, oxide electronics.

\section{INTRODUCTION}

$\mathrm{T}$ RANSITION metal oxides (TMO) are key materials of oxide nanoelectronics [1]. Manganese dioxide $\mathrm{MnO}_{2}$, a representative of the TMO family, is one of the most attractive inorganic materials because of its wide range of applications. In particular, nanostructured $\mathrm{MnO}_{2}$ has received a great attention due to its high specific surface area, functional properties, and potential applications as molecular and ion selective membranes, in capacitors, Li-

Manuscript received November 25, 2013; accepted December 10, 2013. Date of online publication: December 15, 2013.

This work was supported by the Strategic Development Program of Petrozavodsk State University (2012-2016), Ministry of Education and Science of Russian Federation "Scientific and Educational Community of Innovation Russia (2009-2013)" Program through contracts no. 14.740.11.0895, no. 14.740.11.0137, no. 14.740.11.1157, no. 16.740.11.0562, and no. 14.B37.21.0755, as well as "Development of Scientific Potential of High School" Program, contracts no. 2.3282.2011 and no. 2.2774.2011.

V. P. Malinenko is with the Department of Solid State Physics, Faculty of Physical Engineering of Petrozavodsk State University, 185910 Petrozavodsk, Russia (e-mail: vmal@psu.karelia.ru).

L. A. Aleshina is with the Department of Solid State Physics, Faculty of Physical Engineering of Petrozavodsk State University, 185910 Petrozavodsk, Russia (e-mail: aleshina@psu.karelia.ru).

A. L. Pergament is with the Department of Information Measuring Systems and Physical Electronics, Faculty of Physical Engineering of Petrozavodsk State University, 185910 Petrozavodsk, Russia (e-mail: aperg@psu.karelia.ru).

G. V. Germak received his MS degree from the Department of Solid State Physics, Faculty of Physical Engineering of Petrozavodsk State University, 185910 Petrozavodsk, Russia (e-mail: ggv4949@gmail.com). ion batteries, and catalysts [2]. Originally, $\mathrm{MnO}_{2}$ has been widely used as a cathode material for oxide-semiconductor capacitors based on tantalum, niobium and aluminum oxides, and the most important properties of the material for this application are its high conductivity and ability to thermal phase transition into the lower valence states accompanied by evolution of atomic oxygen, while the resistance increases by 3-4 orders of magnitude (see, e.g. [3] and references therein). Much attention is currently paid to various other properties of $\mathrm{MnO}_{2}$, such as, for example, the electrochromic effect [4], thermopower wave phenomenon [5], supercapacitive behavior [6], memory and threshold switching [3], [7], [8]. Also, there are a number of papers [9]-[11] describing an anomaly in the $\mathrm{MnO}_{2}$ conductivity near the Neel temperature which is shown to be associated with the metal-insulator transition [3]. That is why the electrical properties of $\mathrm{MnO}_{2}$ are of especial scientific interest and applied importance.

The most common methods for manganese oxide preparation are the pyrolytic decomposition of manganese nitrate, electrochemical method, hydrothermal synthesis in a magnetic field, and chemical deposition [3], [12]-[15]. Due to a wide range of possible valence states of cations, the structure and phase composition and of manganese oxides are determined by the synthesis conditions and process temperature. Manganese dioxide has many crystallographic forms $\left(\alpha-, \beta-, \gamma-, \delta-, \varepsilon-\right.$ and $\left.\lambda-\mathrm{MnO}_{2}\right)$ that connect $\mathrm{MnO}_{6}$ octahedron units to each other in different ways and have different characters of spatial alternation of the octahedra filled by Mn atoms and empty octahedra [2], [3], [16]-[20].

The crystal structure of the $\beta-\mathrm{MnO}_{2}$ phase (Fig. 1) is a tetragonal modification of the rutile structural type, $P 4 / m n m$ symmetry group, which represents a slightly distorted close-packed hexagonal lattice of oxygen ions [19]. Filled octahedra are bound by two common edges in columns oriented along the $c$ axis, and octahedra of adjacent columns are bound by their vertices (Fig. 1, c). Manganese atoms are arranged in positions 2(a) (000), and oxygen - in positions $4(f)(x x 0)$. Because there are vacancies in the oxygen packing, the formula unit can be written as $\mathrm{MnO}_{2-\mathrm{y}}$ where the non-stoichiometry index is in the range $y=0.01-0.10$ depending on the synthesis method [19]. 

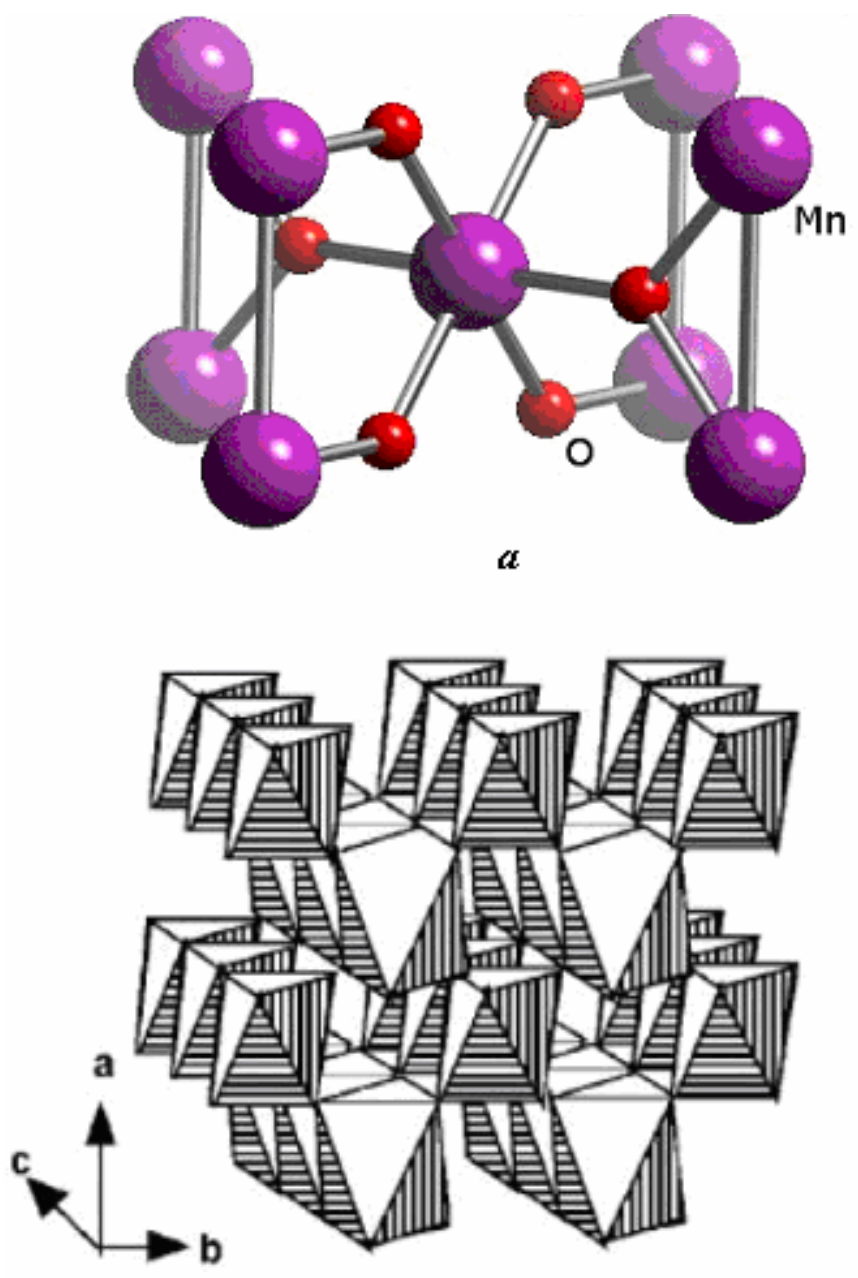

$b$

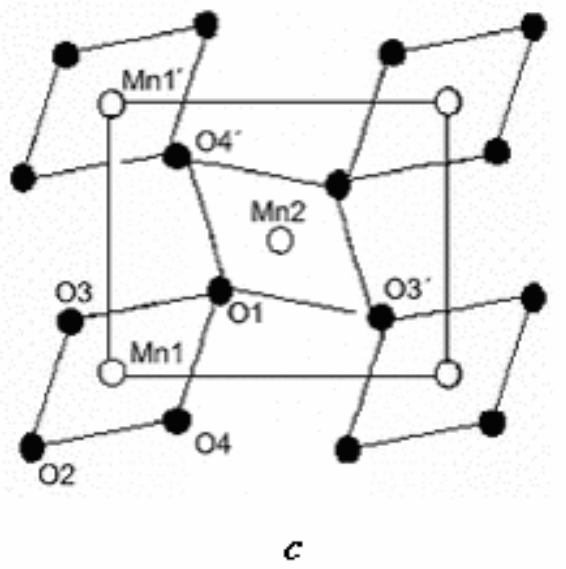

Fig. 1. Crystal structure of manganese dioxide $(a), \mathrm{MnO}_{6}$ octahedron connection in $\beta-\mathrm{MnO}_{2}(b)$ [16]-[20], and projection of octahedron binding onto the $a b$ plane [19].

As to the lower manganese oxides, $\mathrm{MnO}$ and $\mathrm{Mn}_{3} \mathrm{O}_{4}$ are known to be the Mott insulators with relatively high resistivity [16], and $\mathrm{Mn}_{2} \mathrm{O}_{3}$ shows p-type semiconducting properties. $\mathrm{MnO}_{2}$ is an n-type semiconductor and antiferromagnetic with the Neel temperature $T_{\mathrm{N}}=92.5 \mathrm{~K}$ [17]. Conductivity of manganese dioxide is higher than that of $\mathrm{MnO}$ and $\mathrm{Mn}_{2} \mathrm{O}_{3}$ by several orders of magnitude. At temperatures above $723 \mathrm{~K}$, manganese dioxide losses oxygen and transforms into $\mathrm{Mn}_{2} \mathrm{O}_{3}$, which is accompanied by the transformation of the crystalline structure from the tetragonal crystal system of the rutile type into the complex large-period cubic lattice $(a=9.4 \AA)$ and a large number of formula units per unit cell [3], [18], [19].

In this work we have studied the electrical properties of manganese dioxide, particularly, the nature of the $\beta-\mathrm{MnO}_{2}$ conductivity at low temperatures, in its interrelation with caracteristic features of the material crystalline structure, and the metal-insulator phase transition in this compound as well. The effect of electrical switching with current-voltage characteristics of both $\mathrm{N}$ - and S-type has been found in thin-film metal-oxide-metal (MOM) structures on the basis of manganese oxides. Possible mechanisms of switching in terms of the metal-insulator transition (MIT) and thermistor effects are discussed.

\section{EXPERIMENTAL DETAILS}

Thin films of manganese dioxide were deposited by means of thermal evaporation onto glass substrates, covered by a $\mathrm{SnO}_{2}$ layer, using a VUP-5 vacuum setup; the film thickness was $d=250 \mathrm{~nm}$. Also, thin films of manganese oxide were obtained by electrochemical oxidation of metal $\mathrm{Mn}$ in the $\mathrm{KNO}_{3}-\mathrm{NaNO}_{3}$ eutectic melt [21] at $T=600 \mathrm{~K}$ at a constant voltage of about $2 \mathrm{~V}$ for $3 \mathrm{~min}$; the current density diminished from 200 to $100 \mathrm{~mA} / \mathrm{cm}^{2}$ during the oxidation process. For anodic oxide films on $\mathrm{Mn}$, the value of $d$ was $\sim 100 \mathrm{~nm}$. Bulk samples of manganese dioxide were obtained by multiple decomposition (pyrolysis) of manganese nitrate at $T=670 \mathrm{~K}$ on a glass-ceramic substrate or on a tantalum sheet metal with the subsequent separation from the substrate [18], [19]. The total coating thickness was about $1 \mathrm{~mm}$.

The manganese oxide samples were characterized by $\mathrm{X}$ ray diffraction (XRD) using a DRON-6 diffractometer in $\mathrm{CuK}_{\alpha}$ and $\mathrm{FeK}_{\alpha}$ radiation. The characteristics of the atomic structure of pyrolytic manganese dioxide were refined by the full-profile analysis of the XRD patterns of polycrystalline samples [19]. The resistivity of pyrolytic $\mathrm{MnO}_{2}$ was measured in the temperature range 15 to $300 \mathrm{~K}$ by means of a four-probe technique, both $\mathrm{DC}$ and $\mathrm{AC}$ (in a frequency range from 50 to $10^{4} \mathrm{~Hz}$ ), in a GiffordMcMahon cycle cryorefrigerator [3]. We also measured electrical conductivity in the range $T=293-423 \mathrm{~K}$, the Hall effect in the magnetic field of $2.3 \mathrm{~T}$, and the Seebeck coefficient (thermo-electromotive force, thermo-emf). 
The dynamic current-voltage $(I-V)$ characteristics of the thin-film MOM structures were measured by oscillographic method using a sinusoidal signal. The "upper" electrode of the MOM structure was a spring-loaded Au contact, and the "bottom" one was either metal $\mathrm{Mn}$ (in case of anodic oxide films) or conductive oxide $\mathrm{SnO}_{2}$ (in case of vacuumdeposited films). To measure the $I-V$ curves, a digital OWON PDS 5022S oscilloscope and an AC generator were used. The latter was connected in series with the MOM structure and with a $5 \mathrm{M} \Omega$ variable load resistor. The voltage drop across the structure was measured directly as the " $\mathrm{X}$ " input oscilloscope signal, and the current was monitored by determining the voltage drop across an additional series resistor of 1 to $50 \Omega$ as the "Y" input signal.

\section{RESULTS AND DISCUSSION}

\section{A. Crystal Structure and Oxygen Stoichiometry}

The XRD patterns of the manganese oxide samples are presented in Figures 2 and 3. The vacuum-deposited films are amorphous and represent a mixture of $\beta$ - and $\gamma-\mathrm{MnO}_{2}$ (Fig. 3). The results of the full-profile analysis show that the pyrolitic $\mathrm{MnO}_{2}$ samples (Fig. 2) constitute of the finely dispersed $\beta-\mathrm{MnO}_{2}$ phase with the traces of $\gamma-\mathrm{MnO}_{2}, \varepsilon$ $\mathrm{MnO}_{2}$ and $\mathrm{Mn}_{2} \mathrm{O}_{3}$ phases. The periods of the tetragonal unit cell of $\beta-\mathrm{MnO}_{2}$ are $a=b=4.401 \AA$ and $c=2.872 \AA$. The oxygen coordinates in $4 f$ positions are found to be $x=$ 0.302. Calculations of the oxygen-manganese interatomic distances show [19] that the oxygen octahedron is compressed along the (100) direction which connects the octahedron vertices. This deformation results in an increase of the overlap of electron $p$ orbitals of oxygen and $d$ orbitals of manganese and can form the additional splitting of electron levels in the crystal field leading to the appearance of unpaired electrons [20].

The measurements of the conductivity temperature dependence, Hall effect, and thermo-emf show that manganese dioxide is the electron semiconductor with

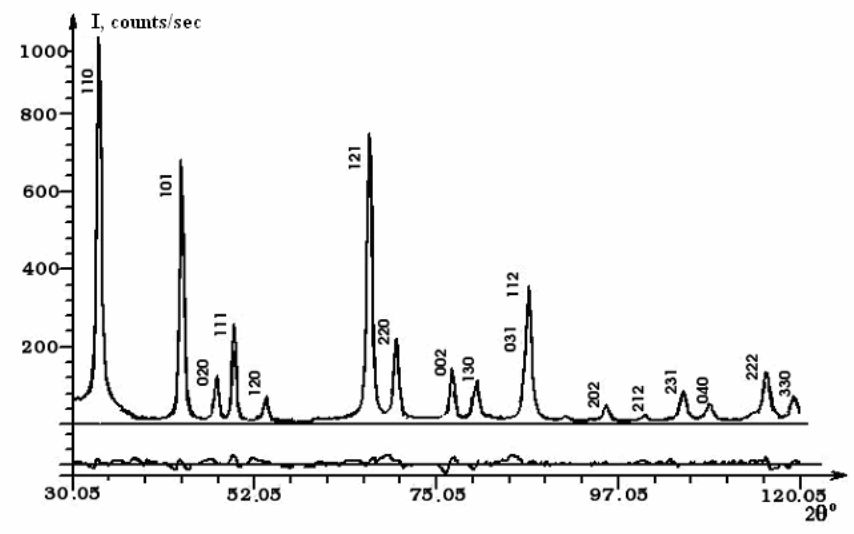

Fig. 2. XRD ( $\mathrm{Cu} \mathrm{K} \mathrm{K}_{\alpha}$ radiation) pattern of pyrolytic $\mathrm{MnO}_{2}$ (upper curve) and the difference between experimental and calculated spectra (lower curve).

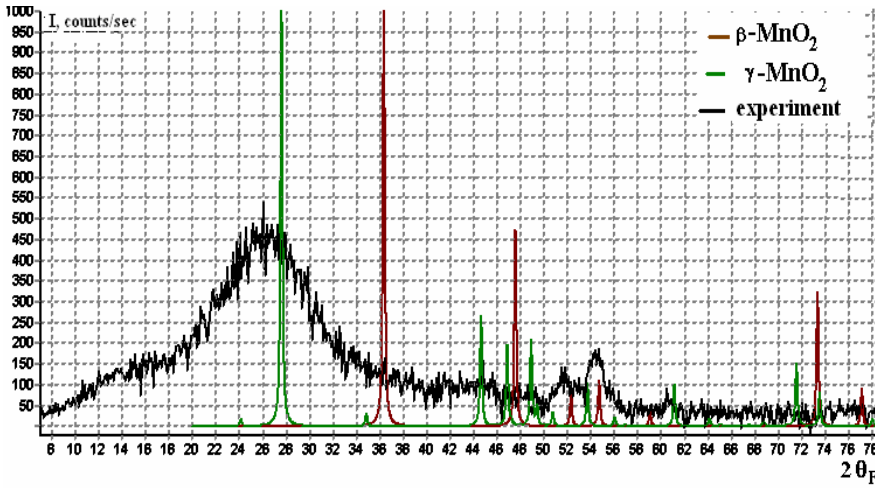

Fig. 3. XRD (Fe $\mathrm{K}_{\alpha}$ radiation) pattern of Mn oxide film and reference spectra of crystalline $\mathrm{MnO}_{2}$ samples.

conductivity of about $1 \Omega^{-1} \mathrm{~cm}^{-1}$ and mobility of $\sim 10$ $\mathrm{cm}^{2} / \mathrm{V} \cdot \mathrm{s}$. The Hall measurements give the carrier density of $\sim 10^{18} \mathrm{~cm}^{-3}$, which is close to the density values in degenerate semiconductors. The activation energy of conductivity is $\sim 0.1 \mathrm{eV}$, and the band gap width equals to 2 $\mathrm{eV}$ [16]. The estimated value of the Fermi energy, from the thermo-emf temperature dependence, is in the range 0.02 to $0.05 \mathrm{eV}$ which also indicates the degenerate character of the electron gas. The nonstoichiometry index determined by the XRD peaks, which are not contributed by the manganese atoms, i.e. by those with the odd sum of indexes, is $y=0.06$. Such nonstoichiometry provides the concentration of trap states of about $10^{17}-10^{18} \mathrm{~cm}^{-3}$, which correlates with the above mentioned Hall-effect-based measurements of electron density.

Such a high vacancy concentration allows us to consider the nonstoichiometric $\beta-\mathrm{MnO}_{2}$ as a heavily doped semiconductor with a subband of impurity levels, which provides a feasibility of the out-of-band electron transfer at low temperatures. The structural distortions, a high nonstoichiometry and, as a consequence, a sufficiently high concentration of impurity states, cause the density state tails near the band edges similarly to those in heavily doped semiconductors. However, at room and higher temperatures the conduction is determined by the activation mechanism. Thus, the electron structure and a low mobility of charge carriers promote the manifestation of different charge transfer mechanisms in different temperature ranges, namely, both the hopping mechanism and the activation one.

The electron capturing oxygen vacancies create mixed valence stares of manganese cations $\left(\mathrm{Mn}^{3+}-\mathrm{Mn}^{4+}\right)$. Interestingly, the mixed valence of $\mathrm{Mn}$ ions is also characteristic of manganites $\mathrm{Ln}_{1-\mathrm{y}} \mathrm{Sr}_{\mathrm{y}} \mathrm{MnO}_{3}$ [22], where $\mathrm{Ln}$ is $\mathrm{La}$ or another rare-earth element, possessing the effect of colossal magnetoresistance (CMR). In these compounds, the mixed valence of manganese is attained via doping. It had been deemed that holes introduced by strontium are localized at manganese ions, i.e. the $\mathrm{Mn}^{4+}$ ions with concentration $y$ are formed. However, recent X-ray emission studies have shown that both the chemical shift and the exchange splitting of the emission X-ray $\mathrm{Mn} \mathrm{K}_{\beta 1}$ 
line are almost the same in the range $y<0.4-0.5$, as is the case in $\mathrm{Mn}_{2} \mathrm{O}_{3}$, and then they decrease quickly to the values characteristic of $\mathrm{MnO}_{2}$ [23] which suggests that the introduced holes are localized at oxygen atoms in the hole doping region (at relatively low $y$ ) and at manganese atoms in the electron doping region, i.e. at higher $y$ values.

\section{B. Electrical Conductivity}

The temperature dependence of conductivity is presented in Fig. 4. The resistance peak observed in the figure in a temperature range of $80-90 \mathrm{~K}$ is the result of the phase transition of $\beta-\mathrm{MnO}_{2}$ from a semiconducting state to a metal state upon decreasing the temperature to $15 \mathrm{~K}$ [3]. The temperature region of the transition is close to the known Neel transition from the paramagnetic state into the ferromagnetic one for manganese dioxide. The temperature dependence of resistance indicates thus the MIT to occur in $\mathrm{MnO}_{2}$. In the temperature range from 300 to $80 \mathrm{~K}$ (Fig. 4) this dependence does not correspond to the exponential one, yet it fits well to the linear dependence in the Mott coordinates $\ln (R) \sim T^{1 / 4}$, which indicates the hopping conductivity mechanism over the polyvalent states of manganese ions with a distance between the centers of 25 $35 \AA$ [18]. However, in the temperature range from 90 to 15 $\mathrm{K}$, the dependence of $\ln R$ on $\ln T$ is almost linear with the slope of about unity, which is characteristic of the metallic conduction. Thus, the character of conductivity of manganese dioxide indicates the presence of the MIT at $T_{\mathrm{c}}$ $\sim 80 \mathrm{~K}$ (on cooling).

In the low temperature region, manganese dioxide is an

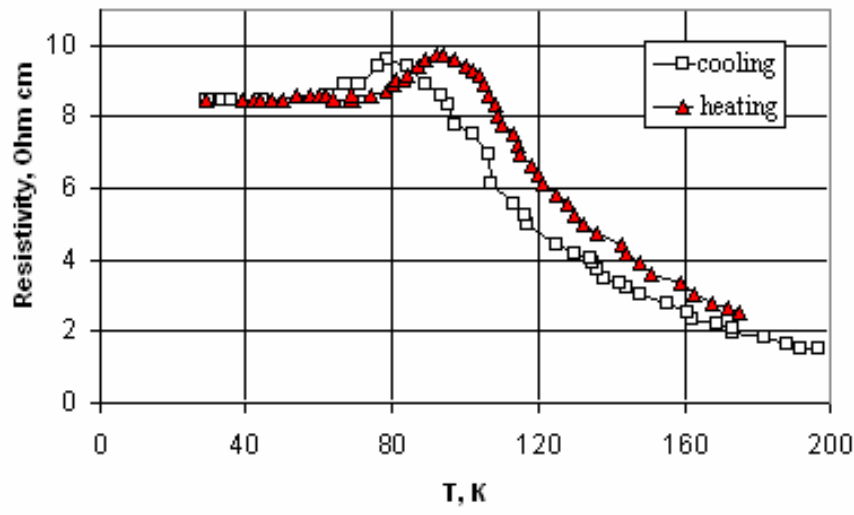

Fig. 4. Temperature dependence of $\mathrm{AC}(70 \mathrm{~Hz})$ resistivity of pyrolytic $\mathrm{MnO}_{2}$.

antiferromagnet with the helical spin ordering [24]. The interaction between the magnetic ions of manganese, which leads to the antiferromagnetic state, occurs through the intermediate interaction with diamagnetic oxygen ions, which weakens the exchange interaction. The conductivity increases probably due to exceeding the energy of the exchange interaction by the thermal energy as the temperature increases, which leads to an increase in the number of unpaired electrons in the paramagnetic state. As the temperature further increases, the conductivity continues to rise, and the Mott hopping mechanism of charge transfer becomes predominant. Similar character of the temperature dependence in ferromagnetic degenerate semiconductors has previously been described [11], [25] for sub-stoichiometric (oxygen-deficient) europium monoxide. The transition from the highly conductive state into the insulating state occurs in such semiconductors upon varying the type of magnetic ordering or its destruction.

For $\mathrm{EuO}_{1-\mathrm{y}}$, lowering the peak magnitude in the $R(T)$ dependence is associated with a decrease in the concentration of impurities (oxygen vacancies). In magnetic semiconductors, the phase transition is associated both with the magnetoelectric effects and with the variation in the electric polarization under the action of the magnetic field at the antiferromagnetic transition [25]. The dielectric constant for manganese dioxide is equal to $\sim 10$, but the effective dielectric constant can decrease near $T_{\mathrm{c}}$ by a factor of several times thereby affecting the mobility of carriers located in the tails of the density of states near the band edge and promoting their delocalization at the phase transition temperature. Manganese dioxide also belongs to this group of substances; however, the reported here phase transition in $\mathrm{MnO}_{2}$ can be also described in terms of the Mott transition [26].

According to the Mott criterion, electrons delocalize if the distance between the atoms $n^{-1 / 3}$ becomes comparable with their atomic radius $a_{\mathrm{A}}$, where $a_{\mathrm{A}}$ is associated with the Bohr radius $a_{\mathrm{B}}=\hbar^{2} \varepsilon / m e^{2}$. The static permittivity $\varepsilon$ is replaced by the effective permittivity, which takes into account the exchange interaction of the spins of conduction electrons with orbital magnetic moments of atoms. At the temperature-induced Mott MIT, the orbital radius $a_{\mathrm{B}}$ decreases near $T=T_{\mathrm{c}}$ thereby providing the fulfillment of the Mott criterion at a given level of impurity centers [25], [26]. The transition from the metallic state into the semiconductor state is possible in such semiconductors as the temperature increases. Thus, the MIT in manganese dioxide is brought about by temperature variation.

In conclusion, we note that the phase transition described above is inverse (re-entrant) MIT, i.e. the metallic phase is low-temperature, while the high-temperature state is insulating. This is rather rare, yet not unique, and unusual phenomenon. In most cases (for example, in vanadium dioxide) the low-temperature phase is insulating, and on heating above a certain temperature $T_{\mathrm{c}}$, the material becomes metallic; for $\mathrm{VO}_{2}$ this transition temperature is $T_{\mathrm{c}}=340 \mathrm{~K}$. The inverse transitions are observed in such compounds as the above-mentioned $\mathrm{EuO}_{1-\mathrm{y}}, \mathrm{NiS}_{2}$ :Se, $\mathrm{Y}_{2} \mathrm{O}_{3-\mathrm{y}}$, and $\mathrm{LaMnO}_{3}$ films [3], [11], [25]-[28]. Also, the high-temperature Mott transition from paramagnetic metal to paramagnetic insulator in $\mathrm{V}_{2} \mathrm{O}_{3}$ : $\mathrm{Cr}$ is inverse with the resistivity peak at $T_{\mathrm{c}} \sim 350 \mathrm{~K}$ for a chromium concentration of around 1 at.\% [26, 29], and in mixed-valence CMRmanganites the sharp resistivity peak has been shown to be caused by the Anderson MIT [22]. 


\section{Switching Effects}

It is known that many TMOs exhibit electrical switching associated with MITs [30]. For the anodic oxide films on $\mathrm{Mn}$, the study of the effect of electrical switching in thinfilm $\mathrm{Mn}-\mathrm{MnO}_{2}-\mathrm{Au}$ sandwich structures showed that the Stype switching was not observed in contrast to, for example, the similar structures based on oxides of vanadium and some other transition metals [30]. Instead, switching with the $\mathrm{N}$-shaped $I-V$ characteristic occurred after electroforming (EF) at $T<80 \mathrm{~K}$ (Fig. 5). In addition, the EF process in the Mn-based structures was hindered, likewise, for example, for the structures based on yttrium oxide [27], i.e., the breakdown took place more often. It should be noted that EF at room temperature always led to breakdown, and the switching effect had never been observed in this case. As was argued in [3], this switching effect was associated with the MIT in $\mathrm{MnO}_{2}$.

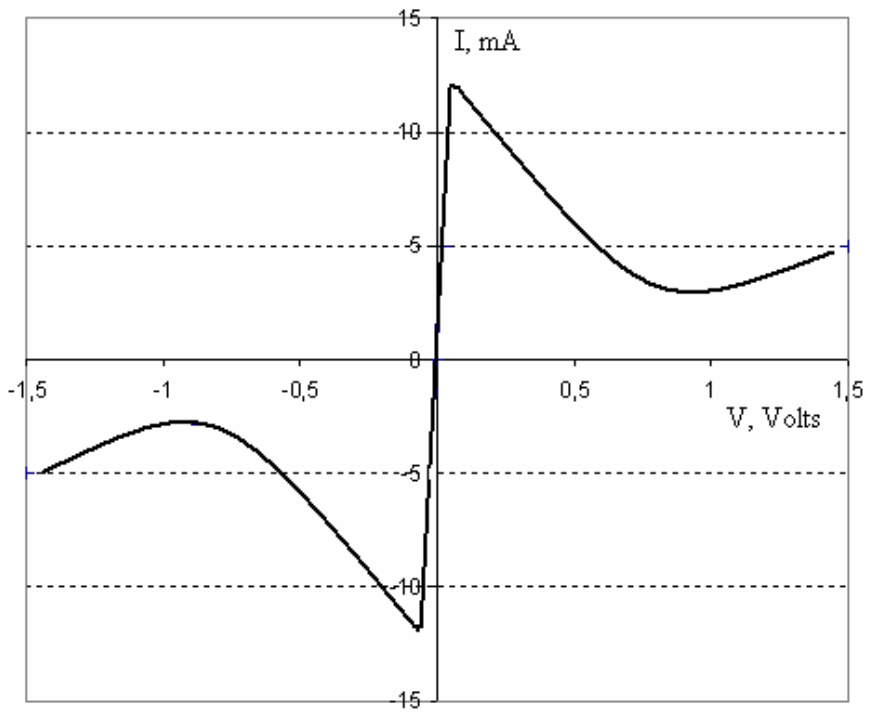

Fig. 5. $I-V$ characteristic of anodic oxide film on manganese at $T=77 \mathrm{~K}$.

On the other hand, in the films obtained by vacuum sputtering, we have observed S-type switching at room temperature (Fig. 6). The threshold voltage is of the order of $1 \mathrm{~V}$ and almost independent of temperature up to $T=375 \mathrm{~K}$

This situation resembles that with yttrium oxide where both S-type and N-type switching has been observed too [27]. One can assume that switching with the S-shaped $I-V$ curves is due to the so-called "thermistor effect". It is well known that the current flowing through a solid generates Joule heat, resulting in an increased temperature. In materials with a negative TCR, a rising temperature induces a current enhancement and an increased power dissipation resulting in a farther temperature increase. This positive electrothermal feedback causes the appearance of a negative differential resistance (NDR) and, accordingly, an $\mathrm{S}$-shaped $I-V$ characteristic [31]. In some cases this process might by modified by high-field effects, such as Schottky injection, impact ionization, Poole-Frenkel effect, Zener or avalanche breakdown. Note that, unlike a conventional thermistor with an Arrhenius-like conductivity law, manganese oxide exhibit a weaker $R(T)$ dependence characteristic of the hopping conductivity mechanism which seems to account for the observed weak temperature

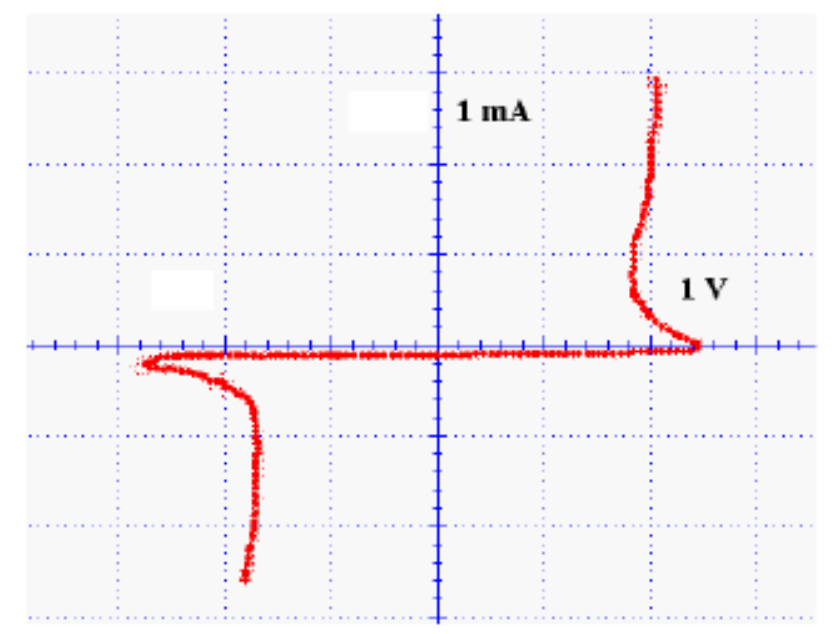

Fig. 6. $I-V$ characteristic of vacuum-deposited $\mathrm{MnO}_{2}$ at $T=293 \mathrm{~K}$.

dependence of the threshold voltage.

An alternative mechanism for the S-type switching effect may be connected with a possible high-temperature usual (i.e. not inverse) MIT in some manganese oxide. As is known [27], [30], EF leads to the formation of a filament of highly conducting material - a switching channel [3]. The phase composition of this switching channel must differ from the material of the initial oxide film, because the channel conductivity exceeds that of an unformed structure by several orders of magnitude. In view of such a plentiful set of phases and crystal structures in the $\mathrm{Mn}-\mathrm{O}$ system, one can suppose that the channel might consist of nonstoichiometric $\beta-\mathrm{MnO}_{2}$, some other polymorphic modifications of manganese dioxide or lower Mn oxides, which might undergo a MIT under the action of either temperature or electric field. However this question obviously requires additional investigation, in particular, measurements of $R(T)$ dependences and switching properties at higher temperatures. If this assumed hightemperature MIT would occur in, e.g., non-stoichiometric $\beta-\mathrm{MnO}_{2}$, one could expect observation of double-NDR $I-V$ characteristics in such samples at low temperatures, analogously to those in $\mathrm{V}_{2} \mathrm{O}_{3}: \mathrm{Cr}$ (Fig. 7) where this effect is due to the MITs [29], with the only difference that in the case of $\mathrm{MnO}_{2}$ this curve should be N-S-shaped (unlike the $\mathrm{S}-\mathrm{N}$-shaped one in Fig. 7), since in chromium doped vanadium sesquioxide the inverse MIT is high-temperature as opposed to $\mathrm{MnO}_{2}$ which, as we discussed above (see Fig. 4), exhibits the inverse MIT at low temperatures.

Note however that the discussed high-temperature MIT in $\beta-\mathrm{MnO}_{2-\mathrm{y}}$ may be unobservable under the temperature change in equilibrium conditions, but display itself only under the applied electric field as the S-type electrical switching effect, because the transition temperature is close 


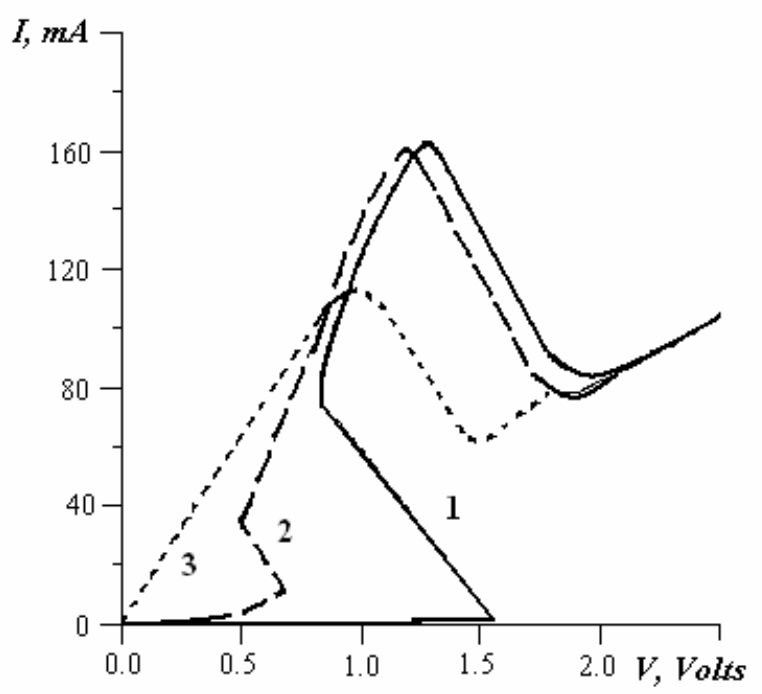

Fig.7. I-V curves with double NDR regions (S-to-N) of vanadium sesquioxide doped with chromium at different temperatures $(1,2,3)$. The switching mechanism is associated with the MITs in $\mathrm{V}_{2} \mathrm{O}_{3}: \mathrm{Cr}$ [29].

to or higher than the temperature of $\mathrm{MnO}_{2}$ decomposition (723 K - see Section I). Such a non-equilibrium transition occurs at switching in those materials where the usual temperature-induced MIT with a well defined $T_{\mathrm{c}}$ does not take place, for example, in chalcogenide glass semiconductors for which the equilibrium transition temperature $T_{\mathrm{c}}$ is higher than $T_{\mathrm{g}}$, while in a high electric field $T_{\mathrm{c}}(E)<T_{\mathrm{g}}$, where $T_{\mathrm{g}}$ is the temperature of the glasscrystal transition and $E$ - the electric field strength [32].

\section{CONCLUSION}

In this work, we have studied the electrical properties (conductivity temperature dependence, Hall and Seebeck effects) of the $\beta$ phase of manganese dioxide obtained by pyrolysis, thermal vacuum evaporation, and anodic oxidation. The change of conductivity mechanisms depending on temperature in a range of 450-25 $\mathrm{K}$ has been shown and the metal-insulator phase transition at low $(<90$ $\mathrm{K})$ temperatures has been revealed. The specific feature of this transition described in terms of the Mott mechanism is that the metal phase exists at low temperatures, while at higher temperatures the material is a semiconductor with the hopping conductivity over the mixed valence states of manganese ions.

The low-temperature switching effect with an NDR and $\mathrm{N}$-shaped current-voltage characteristic associated with the phase transition in manganese dioxide has been found in the MOM two-terminal devices based on anodic Mn oxide films. On the contrary, the devices based on vacuumdeposited films have been found to exhibit an S-type NDR at room and higher temperatures which is suggested to be caused by either the thermistor effect or a high-temperature $\mathrm{MIT}$ in nonstoichiometric $\beta-\mathrm{MnO}_{2}$.
It should be emphasized that the low-temperature MIT in $\beta-\mathrm{MnO}_{2}$ with $T_{\mathrm{c}}=70-80 \mathrm{~K}$ is the inverse Mott transition which is akin, for instance, to the transition in $\mathrm{V}_{2} \mathrm{O}_{3}$ : $\mathrm{Cr}$ at $T_{\mathrm{c}}=190-385 \mathrm{~K}$ (for chromium dopant concentration in the range of $0.5-1.8$ at. \%) [29]. We thus assume that further investigations of the phase transition in manganese dioxide described in this paper is a rather interesting and topical scientific problem, since manganese dioxide can serve as a model object for studying such inverse metal-insulator phase transitions.

Finally, note that the results obtained in the present work indicate the possibility of application of the manganese oxide as a material for thin film sandwich switching devices. Devices with NDR (N- and S-shaped $I-V$ characteristics) have potential applications in microelectronics as switches, memory elements and microsensors. Particularly, manganese oxide microswitches [8] can be utilized for implementation of neuristors built with Mott-transition-based memristors [33] which is one of the rapidly advancing fields of oxide electronics [1], [34].

\section{REFERENCES}

[1] C. Cen, S. Thiel, J. Mannhart, and J. Levy, "Oxide Nanoelectronics on Demand," Science, vol. 323 no. 5917 pp. 1026-1030, Feb. 2009.

[2] W.-H. Ryu, J.-H. Yoon, and H.-S. Kwon, "Morphological control of highly aligned manganese dioxide nanostructure formed by electrodeposition," Materials Letters, vol. 79, pp. 184-187, 2012.

[3] A. L. Pergament, V. P. Malinenko, L. A. Aleshina, and V. V. Kolchigin, "Metal-Insulator Phase Transition and Electrical Switching in Manganese Dioxide," Phys. Solid State, vol. 54, no. 12, pp. 2498-2502, Dec. 2012.

[4] A.L. Gusev, T.N. Kondyrina, V.V. Kursheva, I.A. Pishchurova, O.N. Efimov, S.A. Kondrashov, and A.V. Vanniko, "Perspectives on application of flexible electrochromic panels in housing and communal services facilities and vehicles," Int. Sci. J. Alternative Energy and Ecology, no. 10 (78), pp. 122-137, 2009.

[5] S. Walia, S. Balendhran, P. Yi, D. Yao, S. Zhuiykov, M. Pannirselvam, R. Weber, M. S. Strano, M. Bhaskaran, S. Sriram, and K. Kalantar-zadeh, " $\mathrm{MnO}_{2}$-Based Thermopower Wave Sources with Exceptionally Large Output Voltages," J. Phys. Chem. C, vol. 117, pp. 9137-9142, 2013.

[6] H. Wang, C. Peng, J. Zheng, F. Peng, and H. Yu, "Design, synthesis and the electrochemical performance of $\mathrm{MnO}_{2} / \mathrm{C}_{\circledast} \mathrm{CNT}$ as supercapacitor material," Mat. Res. Bull., vol. 48, pp. 3389-3393, 2013.

[7] M. K. Yang, J.-W. Park, T. K. Ko, and J.-K. Lee, "Bipolar resistive switching behavior in $\mathrm{Ti} / \mathrm{MnO}_{2} / \mathrm{Pt}$ structure for nonvolatile memory devices," Appl. Phys. Lett., vol. 95, art. no. 042105, 2009.

[8] R. Ramesham, T. Daud, A. Moopenn, A. P. Thakoor, and S. K. Khanna, "Manganese oxide microswitch for electronic memory based on neural networks," J. Vacuum Sci. Technol. B, vol. 7, pp. 450-454, 1989.

[9] D. B. Rogers, R. D. Shannon, A. W. Sleight, and J. L. Gillson, "Crystal Chemistry of Metal Dioxides with Rutile-Related Structures," Inorg. Chem., vol. 8, pp.841-850, 1969.

[10] P. H. Klosse, "Electrical Properties of Manganese Dioxide and Manganese Sesquioxide," J. Electrochem. Soc., vol. 117, pp. 854$859,1970$.

[11] E. L. Nagaev, Physics of Magnetic Semiconductors. Nauka, Moscow, 1979, 430 pages [in Russian].

[12] C.-C. Hu and T.-W. Tsou. "Ideal capacitive behaviour of hydrous manganese oxide prepared by anodic deposition," Electrochem. Comm., vol. 4, pp. 105-109, 2002. 
[13] X.-M. Shen and A. Clearfield, "Phase transitions and ion exchange behavior of electrolytically prepared manganese dioxide," J. Solid State Chem., vol. 64, pp. 270-282, Oct. 1986.

[14] H. Y. Kang and C. C. Liang, "The Anodic Oxidation of Manganese Oxides in Alkaline Electrolytes" J. Electrochem. Soc., vol. 115, issue 1, pp. 6-10, 1968.

[15] Y. M. Hu, M. Y. Zhu, Y. Li, H. M. Jin, and Z. Z. Zhu, "MagneticField-Assisted Hydrothermal Growth of Manganese Dioxide Nanostructures and their Phase Transformation," Mater. Sci. Forum vol. 688, pp. 148-152, Jun. 2011.

[16] C. N. R. Rao and B. Raveau, Transition Metal Oxides: Structure, Properties and Synthesis of Ceramics Oxides. Wiley - VCH, NewYork, 1998, 873 pages.

[17] M. Regulski, R. Przeniosło, I. Sosnowska, and J.-U. Hoffmann, "Short and Long Range Magnetic Ordering in $\beta-\mathrm{MnO}_{2}-\mathrm{A}$ Temperature Study," J. Phys. Soc. Jpn., vol. 73, pp. 3444-3447, 2004.

[18] V. P. Malinenko, L. A. Aleshina, S. V. Loginova, and N. D. Tikhonov, "Phase transition in pyrolytic manganese dioxide," in Proc. 10th International Conference on Physics of Dielectrics, St. Petersburg (Herzen State Pedagogical University of Russia), 2004, p. 39.

[19] L. A. Aleshina and S. V. Loginova, "Total Profiling of an X-Ray Pattern of Pyrolytic Manganese Dioxide," Russ. Phys. Journal, vol. 46, Issue 5, pp. 500-503, May 2003.

[20] A. West, Basic Solid State Chemistry. Wiley, New York, 1984.

[21] F. A. Chudnovskii, V. P. Malinenko, A. L. Pergament, and G. B. Stefanovich, "Electrochemical oxidation of $\mathrm{Y}-\mathrm{Ba}-\mathrm{Cu}-\mathrm{O}$ high- $\mathrm{T}_{\mathrm{c}}$ superconductors," Electrochimica Acta, vol. 43, no. 12-13, pp.17791784, 1998.

[22] L. Sheng, D. Y. Xing, D. N. Sheng, and C. S. Ting, "Metal-insulator transition in the mixed-valence manganites," Phys. Rev. B, vol. 56, no. 12, pp. R7053-R7056, 1997.

[23] A. E. Sovestnov, B. T. Melekh, V. V. Fedorov, and E. V. Fomin, "Xray emission studies of evolution of the electron and spin structures of $\mathrm{Mn}$ in mixed manganites $\mathrm{Ln}_{1-x} \mathrm{Sr}_{x} \mathrm{MnO}_{3}(\mathrm{Ln}=\mathrm{La}, \mathrm{Sm}$, and $\mathrm{Ce})$," Phys. Solid State, vol. 54, pp.778-781, Apr. 2012.

[24] E. S. Borovic, V. V. Eremenko, and A. S. Milner. Lectures on Magnetism. Fizmatlit, Moscow, 2005, 510 pages [In Russian].

[25] E. L. Nagaev, "Mott transition in heavily doped magnetic semiconductors," Phys. Solid State, vol. 40, issue 3, pp. 396-399, 1998.

[26] N. F. Mott, Metal-Insulator Transitions ( $2^{\text {nd }}$ edition). Taylor and Francis, London, 1990, 286 pages.

[27] A. L. Pergament, V. P. Malinenko, O. I. Tulubaeva, and L. A. Aleshina, "Electroforming and switching effects in yttrium oxide," Phys. Stat. Solidi (a), vol. 201, no. 7, pp. 1543-1550, 2004.

[28] I. V. Borisenko, M. A. Karpov, and G. A. Ovsyannikov, "Metalinsulator transition in epitaxial films of manganites $\mathrm{LaMnO}_{3}$ grown by magnetron sputtering," Pis'ma v ZhTF [Tech. Phys. Lett.], vol. 39, no. 23, pp. 1-7, Dec. 2013.

[29] F. A. Chudnovskii, A. L. Pergament, G. B. Stefanovich, P. A. Metcalf, and J. M. Honig, "Switching phenomena in chromiumdoped vanadium sesquioxide", J. Appl. Phys., vol. 84, no. 5, pp. 2643-2646, 1998

[30] F. A. Chudnovskii, L. L. Odynets, A. L. Pergament, and G. B. Stefanovich, "Electroforming and switching in oxides of transition metals: The role of metal-insulator transition in the switching mechanism", J. Solid State Chem., vol. 122, pp. 95-99, 1996.

[31] M. Shaw, V. Mitin, E. Schöll, and H. Grubin, The Physics of Instabilities in Solid State Electron Devices, Plenum Press, New York, 1992.

[32] A. L. Pergament, P. P. Boriskov, A. A. Velichko, and N. A. Kuldin, "Switching effect and the metal-insulator transition in electric field," J. Phys. Chem. Solids, vol. 71, pp. 874-879, 2010.

[33] M. D. Pickett, G. Medeiros-Ribeiro, and R. S. Williams, "A scalable neuristor built with Mott memristors," Nature Mat., vol. 12, pp. 414417, Feb. 2013.

[34] S. D. Ha and S. Ramanathan "Adaptive oxide electronics: A review," J. Appl. Phys., vol. 110, art. no. 071101, Oct. 2011. 